\title{
FORTIFY METHOD OF MOVING OBJECT DETECTION BASED ON COLOR AND EDGE GEOMETRICAL FEATURES
}

Ying Lin ${ }^{1,2}$, Yang $\mathrm{Fu}^{1}$, Yueheng Sun ${ }^{2, *}$, Yanghong Sun ${ }^{1}$

${ }^{I}$ School of Management, Chongqing Jiao Tong University, Chongqing, P. R. China 400074

${ }^{2}$ School of Computer Science and Technology, Tianjin University, Tianjin, P. R. China 300072

" Corresponding author, Address: School of Computer Science and Technology, Tianjin University, Tianjin, China, 300072,Tel: +86-22-27401016,Email: yhs@tju.edu.cn

Abstract: A Fortify method of moving object detection is proposed in this paper. Based on color and edge geometric features, the new method can automatically organize a supervised area. First, by the color features in a real supervised scene, the method extracts several regions of interest (ROI) with noise, then matches them with geometric shape by Fourier descriptors in the database and sequentially achieves an automatic-organizing supervised area. Experimental results show that this method has low operation cost, high efficiency, strong anti-jamming, high accuracy and robustness.

Keywords: Fortify method, moving object detection, geometric features, color features

\section{INTRODUCTION}

Along with the computer science and digital image processing technology developing, intelligent video surveillance system has been in intelligent transport, social security, intelligent buildings, grain monitoring and other digital video surveillance areas be widely implied. The most signified point of intelligent video surveillance system is the motion detection and alarm technology. At present the main concentrated of motion detection focus on splitting off the object from the background in the designated area. There are

Please use the following format when citing this chapter:

Lin, Y., Fu, Y., Sun, Y. and Sun, Y., 2009, in IFIP International Federation for Information Processing, Volume 294, Computer and Computing Technologies in Agriculture II, Volume 2, eds. D. Li, Z. Chunjiang, (Boston: Springer), pp. 1135-1143. 
three methods in the general realization of motion detection, as follows: background subtraction; temporal difference; optical flow.

Above are three traditional motion detection methods. In the selection of detection region, the traditional approach can not automatically select the characteristic object as the detection region. In the practical applications, usually taking above three traditional motion detection in the surveillance system for the detection motion by the manual setting, it can't meet the need of automatically select of detected region, and the target objects automatic deployment of intelligent request. Also the manual methods can not meet requirements for providing specific measure changes in the certain practical application, it only provides the judgment of whether or not occur.

Especially, when the monitoring scene is complex, manual setting of the detection region maybe wrongly select the non-motion detection region, thereby increasing the additional computing complexity of the motion detection system. To address the above motion detection setting problem, this paper proposed the intelligent motion region setting method by the HSI (Hue, Saturation, Intensity) color space feature model and use the Fourier descriptors to describe the geometrical shape by (Zahn et at., 1972), and combined with the edge match. The methods applied the sensitivity of color space, and it has the nature of rotation, translation and normalization unchanged character of the normalized Fourier descriptor (Persoon et at., 1986) to descript accurately. Experimental results prove that this method has low operation cost, high efficiency, accuracy and strong anti-jamming, robustness.

\section{INTELLIGENT MOTION DETECTED REGIONAL SETTING}

The computer automatically detects the regional segmentation mainly based on the color characteristics of detection region and the geometrical shape characteristics in the setting of detection region. For this purpose, the extraction of image processing as a key step for the image, which including a large number of various features information. Usually in order to carry out the further image analysis, identification, coding in the main part of pretreatment, the accuracy of segmentation is directly impact on the effectiveness of the following process. However, the application of segmentation technology is constrained by the technical conditions, in the field of image recognition applications is mainly implied by the gray image of segmentation. This paper proposed the initial decomposition of the image by color characteristics in the HSI color space, and use the results of decomposition match with the geometrical shape database that described by 
the Fourier descriptors to achieve the motion detection. The method mainly constitutes by the following two parts:

1、Determination of color characteristics region (HSI space) (Wang Shuan et at., 1999)

Firstly, converting the key frame of real image to the hue sub-space and extracting the color feature corresponding the scope of the $\mathrm{H}$ component in the HSI color space, initially identify the regions of ROI in the color image.

2、 The match of geometrical shape characteristic region

The Identified preliminary ROI regions to binarization and using Canny operator to do the edge segmentation, Fourier descriptors to describe the broader of the segmentation, then matching with the geometrical shape database described by Fourier descriptors.

\subsection{Determination of color characteristics region (HSI space)}

Based on the specific color of rectangular benchmark, firstly extract the color from the hue 、 saturation and intensity model (HSI) color space, initially identifying ROI (region of interests). In the actual image acquisition process, from the acquisition of Monitor RGB format for images, since the first need to achieve a conversion to RGB space HSI space Fig.1 (Zhu Minghan et at., 2005).

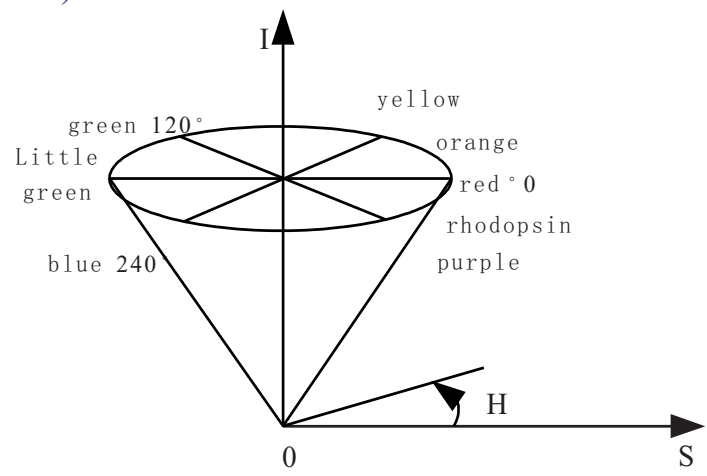

Fig.1. Sketch picture of HIS color model

Firstly will be a normalized: (Lin Ying et at., 2008)

$$
r=\frac{R}{255}, b=\frac{B}{255}, g=\frac{G}{255}
$$

Then proceed into:

$$
H=90-\arctan \left(\frac{F}{\sqrt{3}}\right) \times \frac{180}{\pi}+\{0, g>b ; 180, g<b\}
$$




$$
\mathrm{I}=\frac{\mathrm{r}+\mathrm{b}+\mathrm{g}}{3}
$$

Where:

$$
\begin{aligned}
& S=I-\frac{\min (r, g, b)}{I} \\
& F=\frac{2 r-g-b}{g-b}
\end{aligned}
$$

HIS color model from the diagram, it indicted that we extract any color from the region based on the color model as the actual needs, thus needed to get the color model of regional for the automatic division to a number of the color characteristics of ROI region to binarization and tag.

Then double-edge detection threshold Canny operator (Shridhar et at., 1984)to detect the marginal binarization of ROI region, dual-threshold Canny edge detection operator using different detection threshold can guarantee that the edge of connectivity, traditional threshold method is more vulnerable and the detection edge is not closed, the smaller threshold of detection to repair the edge result of the larger detection threshold, so as to ensure the detected result connectivity and easy identification of detection edge, the specific implementation of the following as shown in Fig.2 :

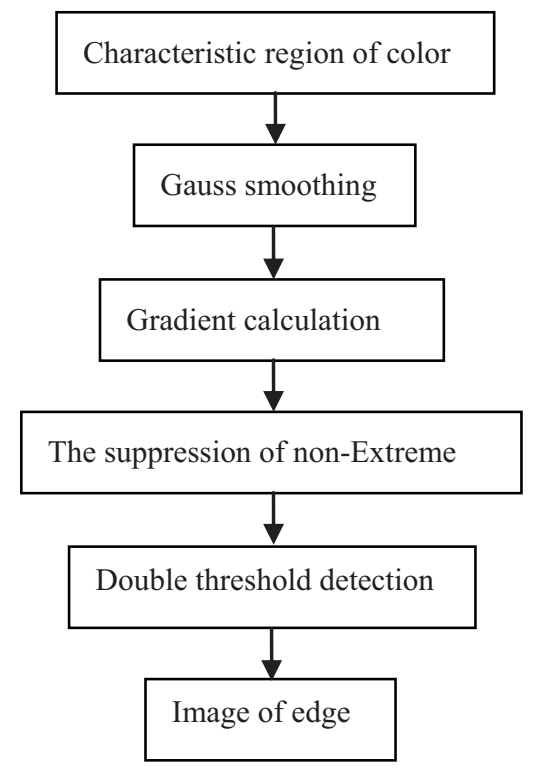

Fig.2: Extract the edge of characteristic region

\subsection{The match of geometry Regional characteristics}

The process of geometrical shape match including the edge extraction, the 


\section{Geometrical Features}

analysis of the relationship of several segment regions in the spatial distribution, with regard to the knowledge of known geometrical shape to analyze and understand the regional segmentation, and these regional characteristics of the region shape extraction through is the basis of the analysis.

First of all, it needs to address the problem how to descript the extracted geometrical shape. This method should meet the description of the same kind of shape has the most similarity, but the different kind of shape has greatest diversity, also should meet the translation, rotation and zoom invariance. In this paper, we take the normalization of shape Fourier descriptors without any relationship to the starting point and the direction to describe the edge. (Rasehom et al.,)

Firstly, we take the extracted region as the complex plane, and the vertical coordinates as the virtual axis, the horizontal coordinates as the real axis. The region on the border point $(\mathrm{x}, \mathrm{y})$ equivalent to the point on the plane $(\mathrm{x}+$ iy), the border point sequence can be written in the complex are as follows:

$$
z_{j}=x_{j}+i y_{j}(j=0,1,2 \cdots N-1)
$$

The definition of Fourier descriptors (FDs) as follows:

$$
\begin{aligned}
& A(k)=\frac{1}{N} \sum_{j=0}^{N-1} Z_{j} \exp \left(-\frac{i 2 \pi k j}{N}\right) \\
& \left(k=-\frac{N}{2}, \cdots,-1,0,1, \cdots, \frac{N}{2}-1\right)
\end{aligned}
$$

Inversion as:

$$
\begin{aligned}
& Z_{j}=\sum_{j=0}^{N-1} A(k) \exp \left(\frac{i 2 \pi k j}{N}\right) \\
& (j=0,1,2, \cdots, N-1)
\end{aligned}
$$

Fast Fourier Transform calculation A (k), followed by the normalization of Fourier descriptors:

$$
N F D(k)=\left\{\begin{array}{l}
0, k=1 ; \\
A(k) / A(1), k=1,2, \cdots, N / 2 ; \\
A(k+N) / A(1), k=-1,-2, \cdots,-N / 2+1 .
\end{array}\right.
$$

A (0) in the tube center of mass, the rest of the Fourier coefficients are complex number. If set the A (0) as 0 , Fourier descriptors has nonrelationship with the location. On the other hand, with the exception of $\mathrm{A}(0)$, the value of the other Fourier coefficient A (1) or A (-1) are largest. Therefore, all coefficients divided by $\|A(1)\|_{\text {or }}\|A(-1)\|_{\text {will normalized }}$ Fourier coefficient between 0 and 1 . If all coefficient divided by the A (1) or 
A (-1), the Fourier descriptors also has non-relationship with the direction and the starting location.

The definition of following Fourier description to do the geometry match:

$$
F F=\frac{\sum_{k=-N / 2+1}^{N / 2}\|N F D(k)\| /|k|}{\sum_{k=-N / 2+1}^{N / 2}\|N F D(k)\|}
$$

Based on the description of the geometrical shape of the Fourier, matching the shape in the geometrical shape database the "shape of feature extraction and the description" of modules, normalized by the Fourier descriptors, the normalization Fourier descriptors to organize the database of geometric shape features by the index.

Through the submission of modules of "the extraction of shape feature and the description", acquired the normalized Fourier descriptors FFi. Do the similarity calculation between FFi and geometrical shape characteristics of the description of FFq, the results of calculation are reflected by the distance between FFi and FFq, the value of the numerical size reflects the degree of similarity between the image to be matched with and the geometric shape database, if those are not matched, then updated the geometric shape database and finished match computing. Fig.3 shows specific matching process (Zhang Kai et al., 2006, Tou et al., 1981):

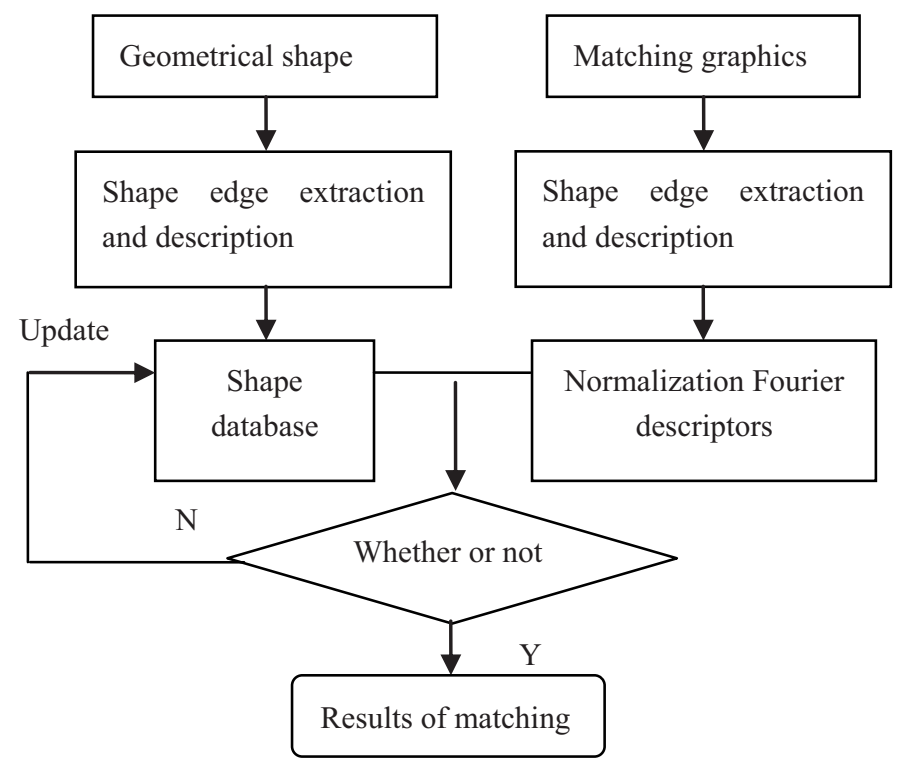

Fig.3: Structure of Shape Feature Match

Through the normalization Fourier descriptors $\operatorname{NFD}(k)$, we can calculate the degree of similarity between any two shapes, and identify the object shape of 
rotation, translation and scale invariant. Because of the frequency components of Fourier transform each other Orthogonal, Euclidean distance to the calculation of a Fourier description of the shape difference, that is:

$$
\text { dist }=\sqrt{\sum_{k=2}^{M}\left\|F F_{i}(k)-F F_{q}(k)\right\|^{2}}
$$

Because the energy of shape is mostly concentrated in the part of lowfrequency, Fourier transform the high frequency components generally smaller and vulnerable to high-frequency noise interference, usually it only uses low-frequency components of normalized Fourier description to calculate the objects' similarity difference.

\section{THE EXPERIMENTAL RESULTS AND ANALYSIS}

Because of the pixel-value of uncompressed $256 \mathrm{bmp}$ image is consistent with the real digital image, so it's suitable for the digital image processing. Therefore, in this paper we take the uncompressed $256 \mathrm{bmp}$ image file format as the experimental image file format.

Applying the designed method to complete the regional selection automatically, based on color and geometrical shape in the real images to select the right region, the correct detection rate is 100 percent, and no misjudged situation occur. The use of color and geometrical shape is the basic characteristic which to complete automatically fortify, from Fig.4 to Fig.5 are the experimental pictures:

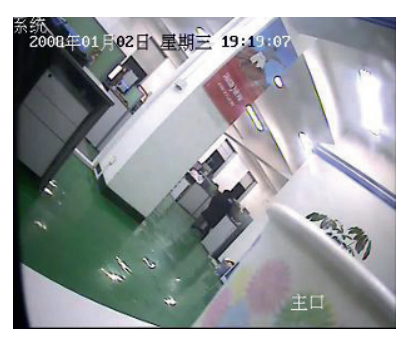

Fig.4. Monitoring Scene

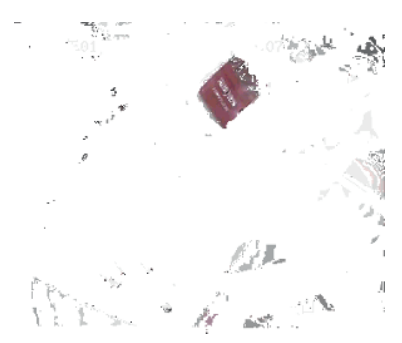

Fig.5. Extract of Color Feature Fig.6. Image of Binarization
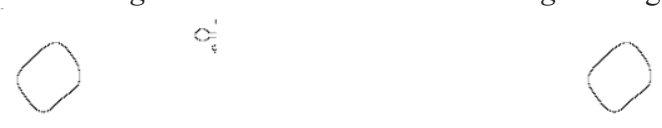
Through the above experiments, we can acquire the following conclusions, with regarding to the fortify method which based on the color and geometrical shape as basic characteristics:

(1) This method used the conversion of HIS color space, and the application of hue in the sub-space made it has good robustness in different lighting conditions, adopting the double threshold Canny edge detection operators to complete ROI edge extraction, the results prove that this method meets the real application requirement.

(2)This method used the concept of ROI, it can adapt to the requirements of real-time monitoring in the supervisory process, and quickly determining the target area, providing the basis for the latter part of the motion detection.

(3)Adopting nature which the same kind of shape has larger similarity, but the different kinds of shape have greater difference. We take Fourier descriptors to describe the geometrical shape, to a certain extent strengthen the system application scope.

\section{CONCLUSION}

From above experiments, we can see that this method adopts the color and the geometrical shape characteristics descript by the Fourier descriptor as the basic conditions to determine the detection region, with characters of high efficiency, low computation, strong anti-jamming and robustness. It provides a new thinking for the intelligent motion detection study, and makes up the additional computing complexity weakness of traditional manual setting method. The experiments show that this method is feasible, also effective, but it can not work for those situations which the objects can't simply distinguished by the colors and geometrical shapes, usually has misjudge phenomenon, so the description of abstract target still needs further study and exploration.

\section{ACKNOWLEDGEMENTS}

This work is supported under the National Natural Science Foundation of China No. 60603027, the Soft Science Research Program of CSTC N0.2007CE9006 and the Bulk Warehouse Grain Quantity Recognition, a project (2007) funded by the Finance Bureau of Chongqing Municipality, P.R. China. 


\section{REFERENCES}

Barron J , Fleet D and Beauchemin S . Performance of optical flow techniques[J]. International Journal of Computer -Vision, 1994, 12(1): 43-77

C T Zahn, R Z Roskies. Fourier descriptors for plane closed curves [J]. IEEE Transactions on Pattern Analysis and Machine Intelligence, 1972, C-21(3): 269-281

Cheng Xiao-chun, a method of shape recognition[J], Pattern Recognition and Artificial Intelligence,2006,6, 126-132(in Chinese)

E, Persoon and K.S. Fu. Shape discrimination using Fourier descriptors[J]. IEEE Trans. on PAMI, 1986, 8(3): 388-397

Gao, J., Zhou, M. \& Wang, H. A Threshold and Region Growing Combined Method for Filament Disapearance Area Detection in Solar Images[C], In Proceedings of The Conference on Information Sciences and Systems. 2001, The John Hopkins University: 243-245

Lin Ying, Fu Yang. The key of bulk warehouse grain quantity recognition ---- Rectangular benchmark image recognition[J].Journal of Zhejiang University(Engineering Science), 2007,41(40): 1643-1646 (in Chinese)

M.D. Kelly. Edge Detection in Pictures by Computer Planning[J], Machine Intelligence, 1973,Vol. 6 (American Elsevier, New York), pp. 397-409

Marr, D. \& Hildreth, E.. Theory of Edge Detection[J], Proceedings of the Royal Society London 1980, B207: 187-217

Shridhar M, Badreldin A. High accuracy character recognition algorithm using Fourier and topological descriptors[J]. Pattern Recognition, 1984, 17(5):515 524

T. Poggio, H. Voorhees and A. Yuille(1985).A Regularized Solution to Edge Detection[J], A. I. Memo 883, M.I.T, 24(3): 23-26

Tou, J.T. \& R.C. Gonzalez. Pattern Recognition Principles[M], Addison-Wesley Publishing, Reading, 1981, MA, USA

Wang Shuan,AI Hai-zhou, HE Ke-zhong. Difference image based multiple motion targets detection and tracking[J]. Journal of Image and Graphics, 1999, 4 (A):471-475(in Chinese)

Zhang Kai, the application of Visual $\mathrm{C}++$ in image processing[J], Internal Science and Technology, 2006,2, 245-252(in Chinese)

Zhang, T.Y. \& C.Y. Suen. A fast parallel algorithm for thinning digital patterns[J], CommACM 1984, 27(3): 236-239

Zhu Minghan, Luo Dayong. Moving Objects Detection Algorithm Based on Two Consecutive Frames Subtraction and Background Subtraction[J]. Computer Measurement \& Control, 2005, 3(6): 215-217(in Chinese) 